\title{
Cloud-based Non-intrusive Load Monitoring System (NILM)
}

\author{
Keh-Kim Kee, Yun Seng Lim, Jianhui Wong, Kein Huat Chua
}

\begin{abstract}
Design and development of a cloud-based non-intrusive load monitoring System (NILM) is presented. It serves for monitoring and disaggregating the aggregated data such as smart metering into appliance-level load information by using cloud computing and machine learning algorithms implemented in cloud. The existing NILM systems are lack of scalability and limited in computing resources (computation and data storage) due to dedicated, closed and proprietary-based characteristics. They are inaccessible to variety of heterogeneity data (electrical and non-electrical data) openly for improving NILM performance. Therefore, this paper proposed a novel cloud-based NILM system to enable collection of these open data for load monitoring and other energy-related services. The collected data such as smart meter or data acquisition unit (DAQ), is pre-processed and uploaded to the cloud platform. A classifier algorithm based on Artificial Neural Network (ANN) is implemented in Azure ML Studio (AzureML), followed by the classifier testing with different combinations of feature set for the performance comparison. Furthermore, a web service is deployed for web APIs (Application Programming Interfaces) of applications such as smart grid and smart cities. The results shows that the ANN classifier for multiclass classification has improved performance with additional features of harmonics, apart from active and reactive powers used. It also demonstrates the feasibility of proposed cloud-based classifier model for load monitoring. Therefore, the proposed solution offers a convenient and cost-effective way of load monitoring via cloud computing technology for smart grid and smart home applications. Further work includes the use of other ML algorithms for classifier, performance analysis, development of cloud-based universal appliance data and use cases.
\end{abstract}

Index Terms: non-intrusive, load monitoring, cloud computing, artificial neural network, application programming interface.

\section{INTRODUCTION}

The combustion of fossil fuel is commonly used by electricity generation plants. The emission of greenhouse gases (GHGs) is always recognised as the main cause of global warming [1]. To mitigate the issue along with the energy crisis, many global public policies are introduced. COP21, also known as the 2015 Paris Climate Conference,

Revised Manuscript Received on September 22, 2019.

Keh-Kim Kee, University College of Technology Sarawak, Sibu, Sarawak, Malaysia.

Yun Seng Lim, Universiti Tunku Abdul Rahman, Sungai Long Campus, Cheras, Selangor, Malaysia.

Jianhui Wong, Universiti Tunku Abdul Rahman, Sungai Long Campus, Cheras, Selangor, Malaysia.

Kein Huat Chua, Universiti Tunku Abdul Rahman, Sungai Long Campus, Cheras, Selangor, Malaysia. mandated the responsibility of each country to reduce both energy consumption and $\mathrm{CO}_{2}$ emission in order to move forward to a low-carbon and sustainable society [2]. However, the mitigation efforts need to be extended to energy efficiency (EE) at consumers (end-users) level, for instance, reduction of energy demand by the adoption of energy demand management.

The buildings sector consumes $36 \%$ of the global energy and emits $39 \%$ of energy-related carbon emission [3]. A study revealed that the buildings with EE strategies have validated an achievement of $74 \%$ of energy saving as compared to traditional buildings [4]. It should be noted that EE policies and projects to conserve energy consumption in buildings require real-time feedback of disaggregated load information to end-users. The survey reported that the energy saving has a potential up to $20 \%$ by incorporating detailed load consumption data feedback to end-users [5].

As the main enabler of these strategies, load monitoring (LM) facilitates valuable feedback consumption data for improved energy-awareness of appliance usage and consumption profile. In general, LM is classified into hardware-centric (intrusive option, known as ILM) and software-defined (non-intrusive option, known as NILM) approaches. Traditional ILM is relatively easier to acquire consumption information of individual appliance but at the expense of complexity in installation and high costs of hardware and labour. On the contrary, NILM is featured with ease of installation and requires almost no maintenance but it is complexity in software algorithms and computation-intensive.

The existing NILM systems are generally lack of scalability and limited computing resources (computation and data storage) due to closed, dedicated and proprietary-based. It is inaccessible to variety of heterogeneity data openly (electrical and non-electrical data) for improved NILM performance. The literature of the use of cloud computing in NILM is still limited with literature gap, to the best of authors' knowledge. This paper aims to propose a cloud-based NILM system for load monitoring based on the aggregated data such as smart metering into appliance-level load information by using cloud computing and machine learning (ML) algorithms implemented in cloud. The rest of this paper is arranged as follows: Section II discusses the literature review related to NILM and its framework, general layout of cloud-based system and ML algorithm based on ANN. The architecture of the proposed system and introduction to Azure ML Studio are described in Section III. The results of the proposed 
system are discussed in Section IV and finally the conclusions are drawn.

\section{LITERATURE REVIEW}

\section{A.Non-intrusive Load Monitoring (NILM)}

The research of NILM was introduced by Hart in early 1980 s who proposed a systematic procedure to apply on a 2-D $\Delta \mathrm{P}-\Delta \mathrm{Q}$ feature plane [6]. The work focused on the monitoring of residential appliances, particularly their operating state, by using aggregated load (i.e. composite signal measured of current and voltage) at the power entry point of the household.

Disaggregation of appliance loads is a mathematical approach to infer the operating status of individual appliances based on electrical measurement data from a smart-meter or dedicated power meter. The disaggregation problem is mathematically formulated. Suppose the sequence of aggregated power consumption $P=\left\{P_{1}, P_{2}, \ldots P_{T}\right\}$ of $\mathrm{N}$ active appliance at $t=\{1,2, \ldots T\}$. The task of NILM algorithm is to infer the power contribution $p_{t}^{i}$ of appliance $i \in\{1,2, \ldots N\}$ at time $\mathrm{t}$, such that at any point in time $\mathrm{t}$,

$P_{t}=\sum_{i=1}^{N}\left(a_{i} p_{t}^{i}\right)+\sigma(t)$

where $a_{i}$ is a Boolean variable represents the operating status of appliance $\mathrm{i}$, and $\sigma(t)$ represents measurement noise and any uncounted contribution of appliances, as shown in Eq.(1).

In general, NILM framework consists of four stages, as depicted in Fig. 1, for load monitoring procedure. The modules include Data Acquisition \& Pre-processing, Event Detection, Feature Extraction and Load Identification [7]. The NILM framework is commonly implemented in offline mode or standalone on-site computing machine (PC). The host PC is running a NILM algorithm for load monitoring and result visualisation on energy dashboard to motivate behaviour change on energy saving by the occupants. However, the model is highly restricted in functionalities due to the limited access of data sources and computing resources. To resolve the problem, cloud technology is incorporated with Internet of Things (IoT), opening an opportunity to leverage the implementation of load monitoring with unlocked services.

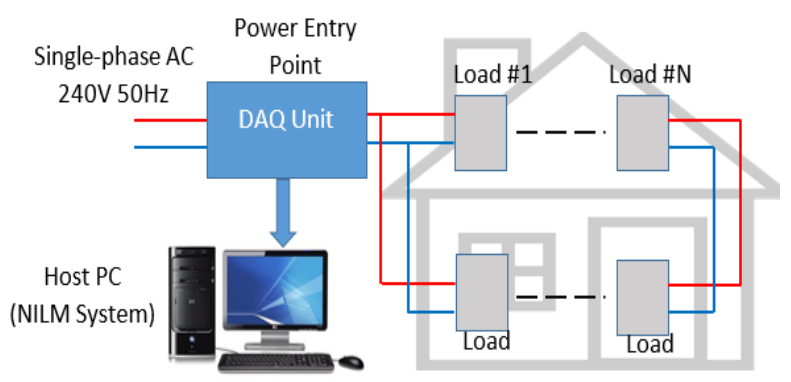

Fig. 1. The layout of typical NILM System

\section{B.Cloud Computing in NILM}

Cloud computing is the methods to allow the end users to connect and gain access into pooling of computing resources (either hardware or/and software) managed by a third party in the Internet "cloud" environment. According to NIST (The National Institute of Standards and Security), it is defined as "a model for enabling ubiquitous, convenient, on-demand network access to a shared pool of configurable computing resources that can be rapidly provisioned and released with minimal management effort or service provider interaction" [8].

Incorporated with Internet-of-Things (IoT), cloud computing is widely used in instrumentation and measurement systems [9], thereby the application software is (1) not hosted on the local machine but on several remote servers connected via internet cloud. These online computing machines are collectively processing the measured data from several measurement devices, and providing relevant energy services to the end users. By deployment of cloud computing in NILM, monitoring of electrical appliance state is accomplished by the platform with large capacity of data storage, high speed of data processing and accessible to wide range of homogeneous or heterogeneous data, to unlock new and improved services to energy stakeholders. For instances, an improved load prediction for smart homes and smart grid applications by using diverse sources of data which include historical data, NILM data, weather and others. Fig. 2 depicts typical architecture layout of cloud computing in NILM. The cloud computing offers an efficient method of data storage on the cloud platform as public data and generic appliance signature database for NILM research. Machine learning algorithms on cloud platform enables the extraction of good insights and deliver better decision support services.

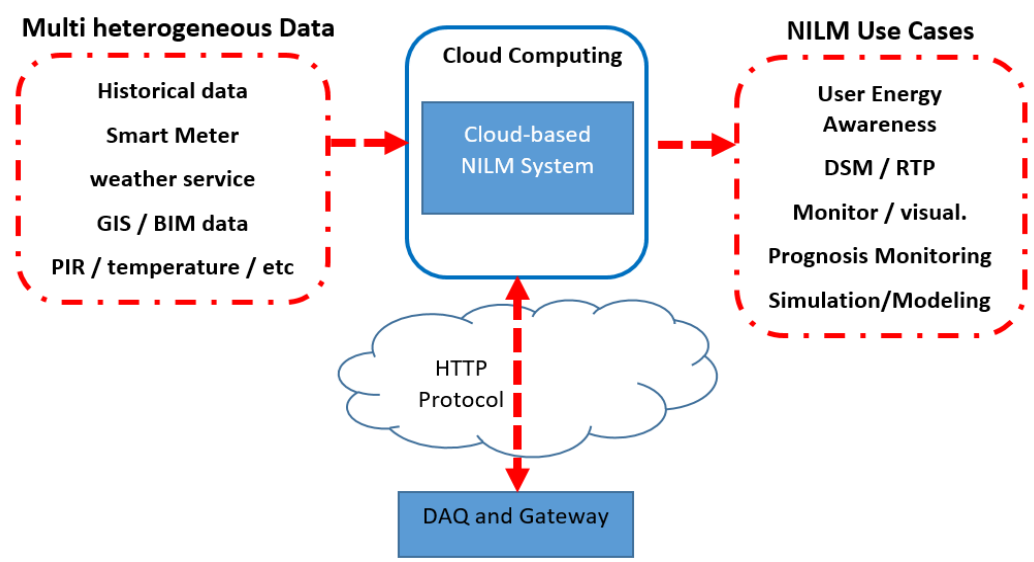

Fig. 2. An architecture of cloud-based NILM system 


\section{C.Machine Learning Algorithm for NILM}

Inspired from the biological nervous system of human brain, artificial neural network (ANN) is essentially a machine learning algorithm which simulates the way of human brain information processing [10]. With the interconnected artificial neurons, ANN can solve various complex problems such as modelling, classification and prediction at high speed. With historical data, ANN performs supervised learning based on dataset of input-output pairs of data involving numerous parameters. For multiclass classification problem, a typical ANN layout is depicted in Fig. 3.

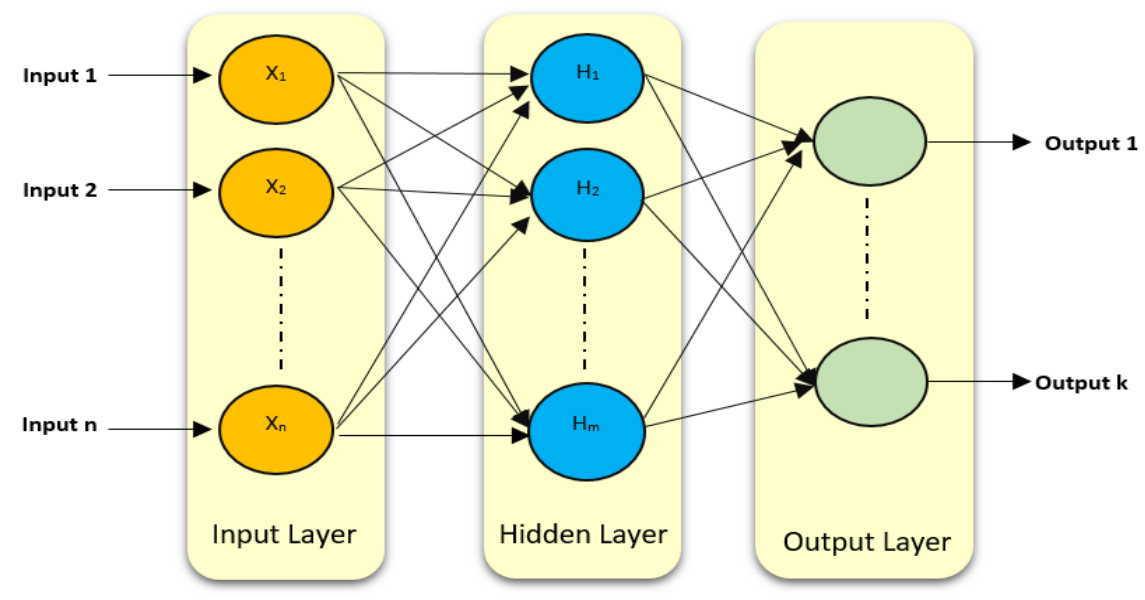

Fig. 3. Typical neural network in multiclass problem recommender, and remote fault diagnosis.

ANN network is typically made up by three layers, i.e. input layer, hidden layer and output layer. The input layer consists of several neurons which is corresponding to the number of input parameters selected [11]. Hidden layer is inserted in between input and output layers, used to perform computations on the weighted inputs which are then applied with activation functions to produce classification output. Generally, the value of each weight is assigned and correlated with the importance of an input feature used to the output layer. In supervised learning, ANN is trained to learn to form a mapping of input features to observed output classes for multiclass classification problem. To further improve performance, a back propagation (BP) algorithm is used to redistribute an error factor from output layer back to the input by re-adjusting of different weights [12].

\section{THE PROPOSED FRAMEWORK OF CLOUD COMPUTING IN NILM}

We present an online appliance classification based on power measurements at $1 \mathrm{~Hz}$ sampling frequency. Cloud technology offers an opportunity to leverage load monitoring services by using cloud resources, such that computational intensive machine algorithms and data analytics can be accomplished efficiently and cost-effectively. The cloud also allows the creation of load pattern library and powerful computation platform. In return, disaggregated load data can be shared online with the stakeholders (utility companies, grid operators, government and consumers). Take for instance, the actionable NILM data will be shared with grid operator and end-users for demand response programs. The heterogeneous data in a multi-modal framework allows application of NILM by combining both power and non-power related data sources. It unlocks several new services such as data analytics, insights, actionable,

\section{A.Proposed System Layout}

The cloud-based NILM system is proposed specifically for load monitoring of electrical appliances based on electrical data uploaded from households. Fig. 4 illustrates the layout of the proposed system which comprises mainly an IoT-based data acquisition unit (i-DAQ) and Cloud Computing Servers (CCS), connected via Internet "cloud".

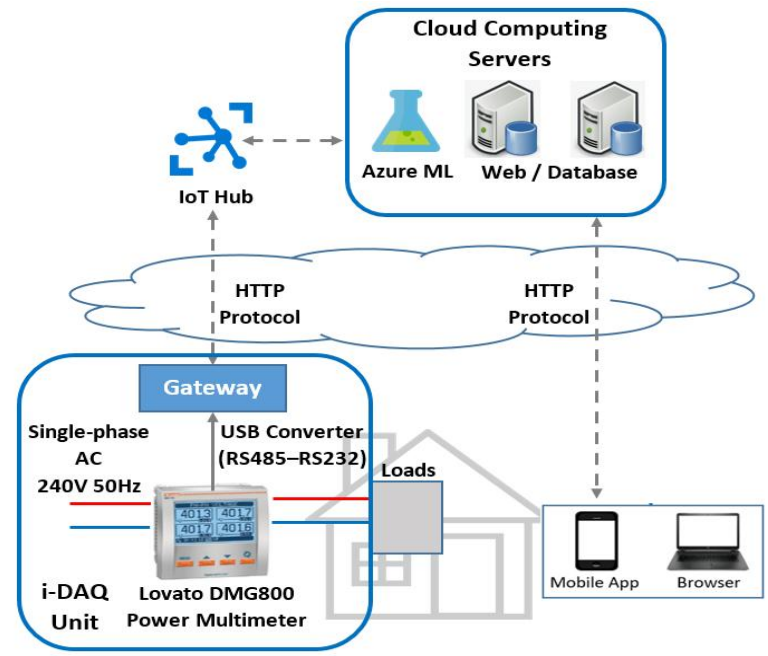

Fig. 4. A proposed layout of Cloud-based NILM system

i-DAQ unit retrieves the power data from energy meter or smart meter, and uploading to CCS via IoT Hub. In our experimental setup, Lovato DMG800 Power Multimeter is used to acquire the relevant power parameters such as real power $(\mathrm{P})$, reactive power $(\mathrm{Q})$ and harmonics data. USB converter is used to convert data signal from RS485 to RS232 so that the acquired data will be uploaded to the Cloud. In CCS, several services include web server, data storage, Azure ML Studio (AzureML) and

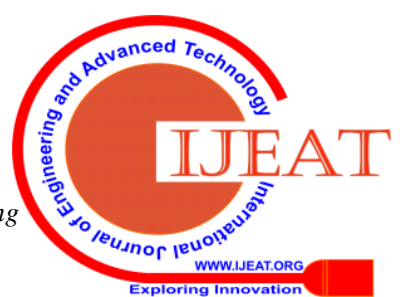


access of heterogeneous data, are used for data computation and information sharing and intelligent control.

\section{B.Dataset}

The dataset consists of actual readings of electrical parameters of the selected electrical appliances. The readings are acquired from $\mathrm{i}-\mathrm{DAQ}$ Unit at a sampling rate of $1 \mathrm{~Hz}$. Each load device has 200 measurement for electrical parameters. There are six load devices for the experimentation setup, namely incandescent light bulb (Bulb), hair dryer (Dryer), selected power electronic device (PE), PC, Fluorescent Light (FL) and Compact Fluorescent Light (CFL). Table I illustrated electrical parameters of the analysed loads with mean and standard deviation values.

Table- I: Electrical parameters of the analysed loads

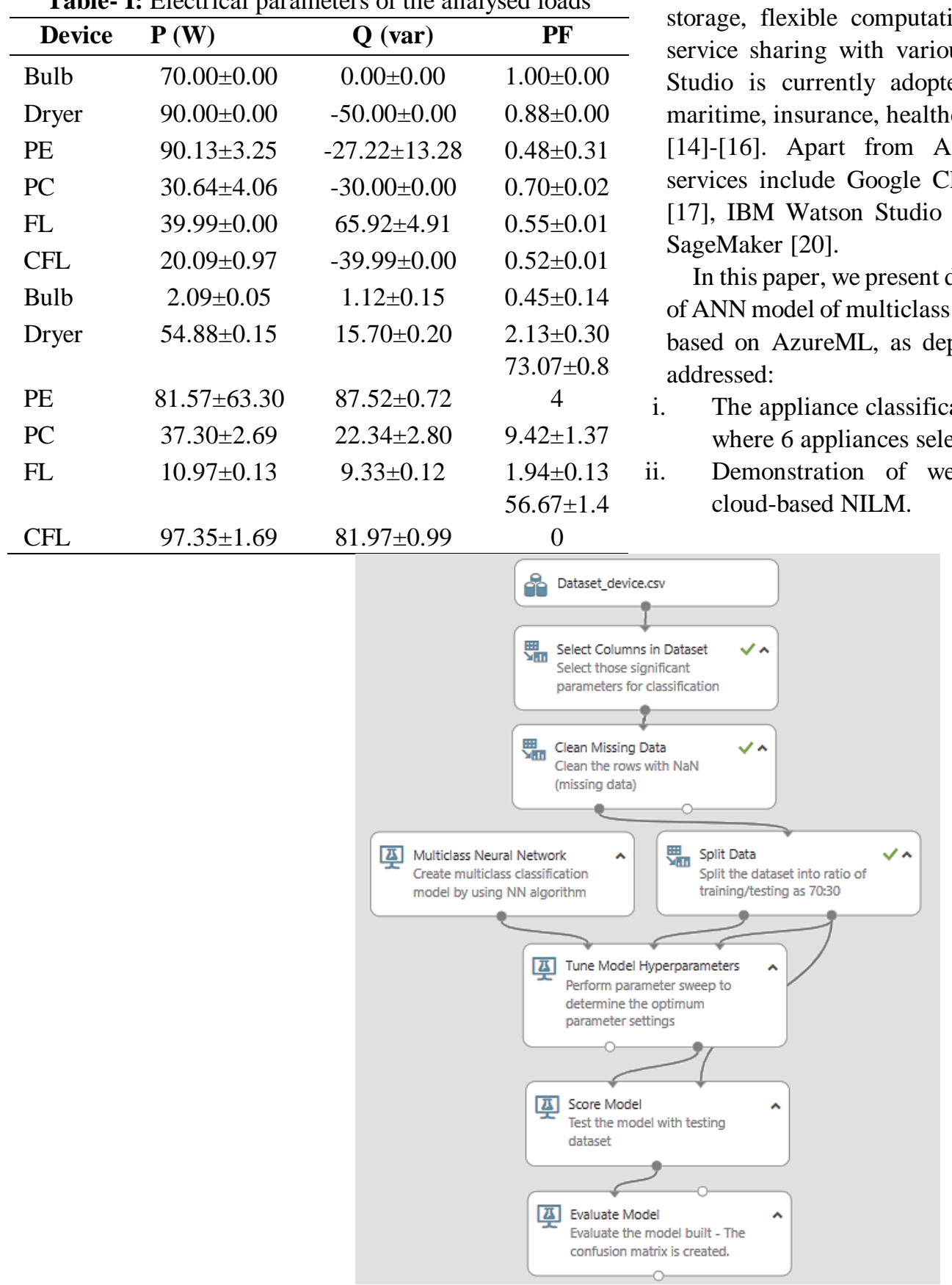

Fig. 5. Implementation of ANN-based multiclass classification on AzureML 
It is important to perform data pre-processing of available data prior the implementation of ML algorithm. It aims to capture substantial characteristics of parameters as the features for further processing. It is generally involving dataq adjusting or cleaning of missing (null) data by replacing with other value or by removing them entirely. Tune Model Hyperparameters module is used to determine optimum hyperparameters for a given ML model. It builds and tests multiple models, using different combinations of setting, and compares metrics over all models to get the combination of settings.

\section{RESULTS AND DISCUSSION}

Multiclass Neural Network algorithm is embedded into NILM system to identify actual loads by using dataset obtained from i-DAQ unit from household. The dataset is used as input features for training and validation. Firstly, we evaluate performance of the proposed system with different combinations of input features to point out the accuracy level of load classification and identification task. Secondly, the web service on Azure is implemented for appliance classification.

Confusion matrix is used as the performance metric of NILM system. Each row of matrix represents the instances of an actual class and each column represents the instances of a predicted class. The identification accuracy of the respective class is indicated by the diagonal values, as highlighted in Fig. 6. However, the row values in parallel illustrate that a particular class is wrongly predicted with other class.

\section{A.Multiclass NN Classifier - single feature used, real power (P)}

For the first case, single real power $\mathrm{P}$ is selected as the input feature for training and validation. The confusion matrix as depicted in Fig. 6, has overall accuracy of $82.94 \%$, which means an error rate of $17.06 \%$. As it is evident classes Bulb and CFL are identified with high accuracy rate of $100 \%$. The result also shows the confusion of input feature to classify between (1) PC and FL (2) Dryer and PE due to difficulty in discriminating caused by the overlapping of input feature used.

\section{Metrics}

Overall accuracy

0.829412

Average accuracy

Micro-averaged precision

0.829412

Macro-averaged precision

$\mathrm{NaN}$

Micro-averaged recall

Macro-averaged recall

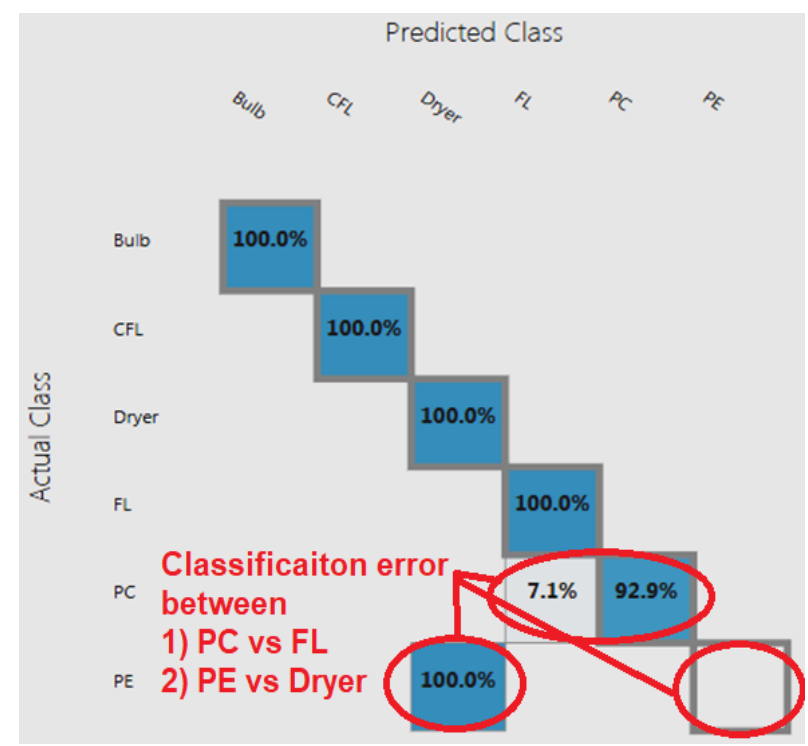

Fig. 6. Confusion Matrix of Multiclass NN Classifier with P feature.

\section{B.Multiclass NN Classifier - two features used, real power $(P)$ and reactive power $(Q)$}

In this case, both real power $\mathrm{P}$ and reactive power $\mathrm{Q}$ are used as the input features for training and validation. The confusion matrix as depicted in Fig. 7, shows an improved overall accuracy of 0.841 . However, the result also shows $100 \%$ wrong classification between PE and Dryer, due to overlapping of features $\mathrm{P}$ and Q used for both appliances.

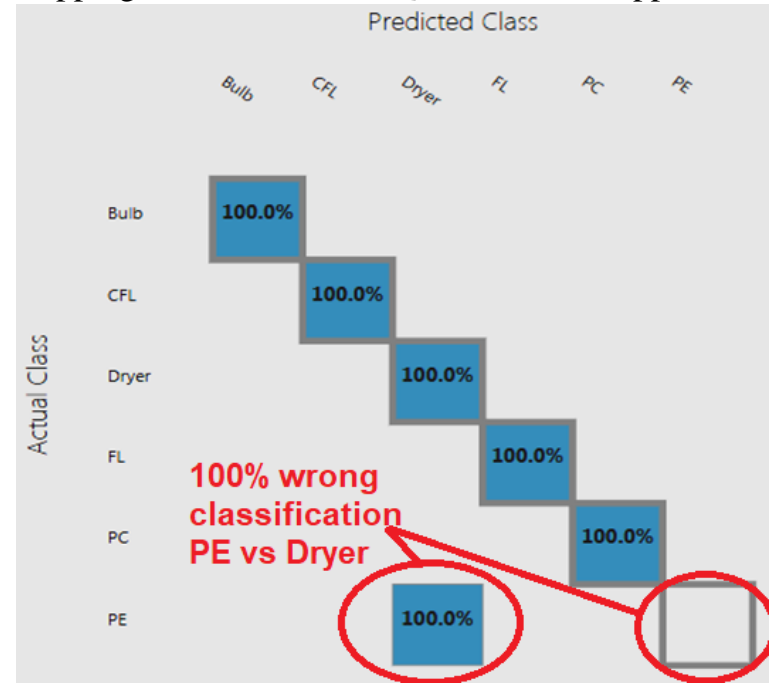

Fig. 7. Confusion Matrix of Multiclass NN Classifier with $P$ and $\mathrm{Q}$ features.

\section{C.Multiclass NN Classifier - powers $P$ \& Q, and odd current harmonics}

For third case, real power $\mathrm{P}$, reactive power $\mathrm{Q}, 3^{\text {rd }} \mathrm{Har}, 5^{\text {th }}$ Har (addition of current harmonics parameters) are selected as the input features for training and validation. By additional input features i.e. odd current harmonics, ANN has successfully classified all the load appliances, as depicted in Fig. 8. It can be noted that PE and Dryer have similar PQ properties, but different harmonics due to non-linear load. Hence, the overlapping of power features in previous cases can be overcome by additional signature features such as 
current harmonics for improved classification performance.

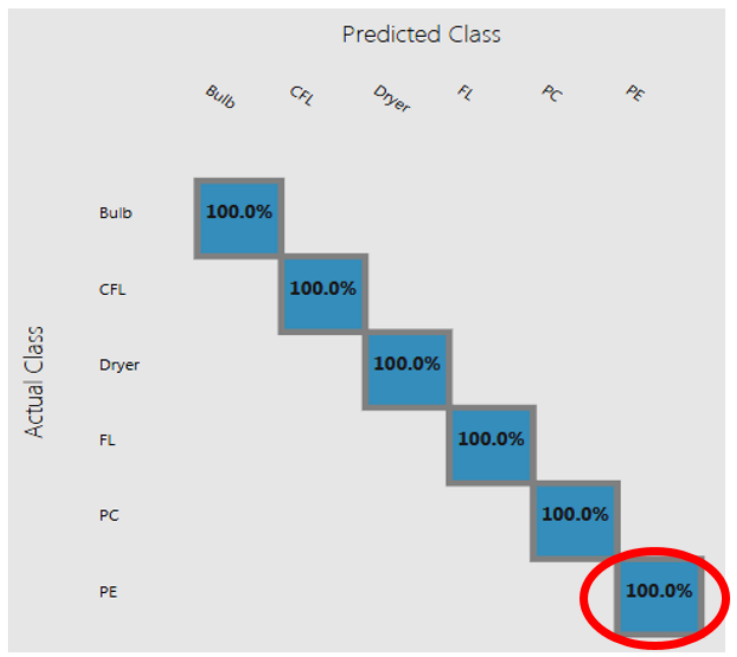

Fig. 8. Confusion Matrix of Multiclass NN Classifier with $P$, $\mathrm{Q}$ and odd current harmonics features

\section{D.Web Services and API Service Call}

The classification model can be deployed as web service on Azure through two types of services, i.e. Request-Response Service (RRS) and Batch Execution Service (BES). RRS is used for lower latency, highly scalable service that provides an interface to the stateless models created and deployed from the Azure ML Studio. The API service call is made by using designated API key. As illustrated in Fig. 9, the classification model is modified by the addition of web service input and output modules. To access the web service, user data is entered through the Input Module and scored by Score Model Module. In return, the classification results are feedback to the user through Web Service Output Module, as depicted in Fig. 10.

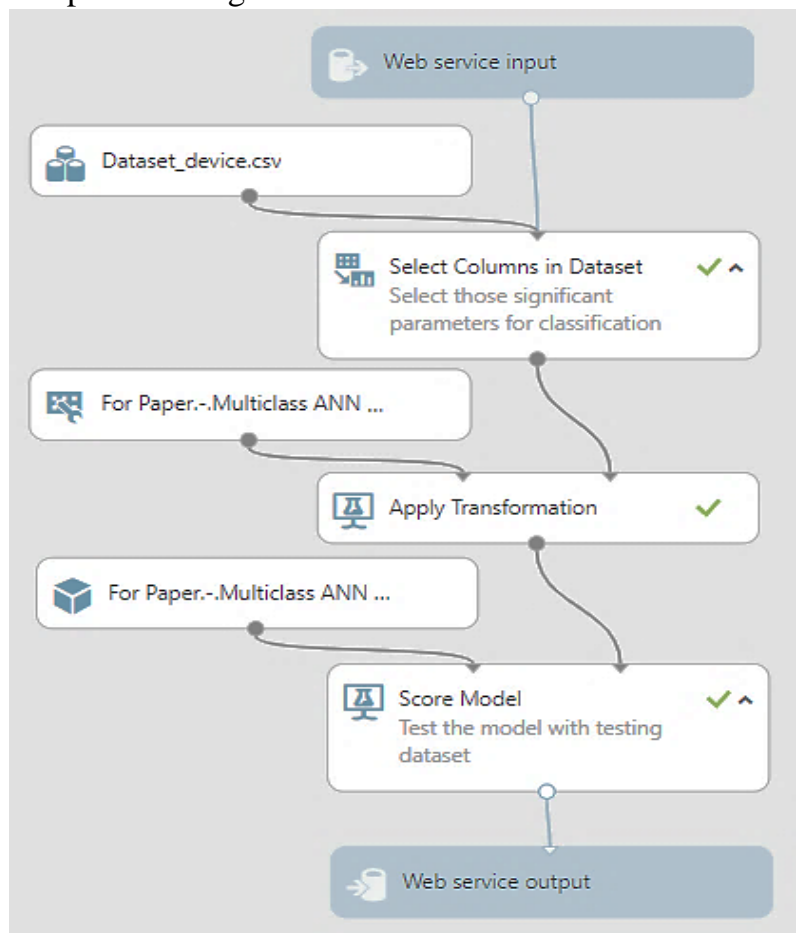

Fig. 9. Web Service Module based on Multiclass Classification modelling in AzureML Studio

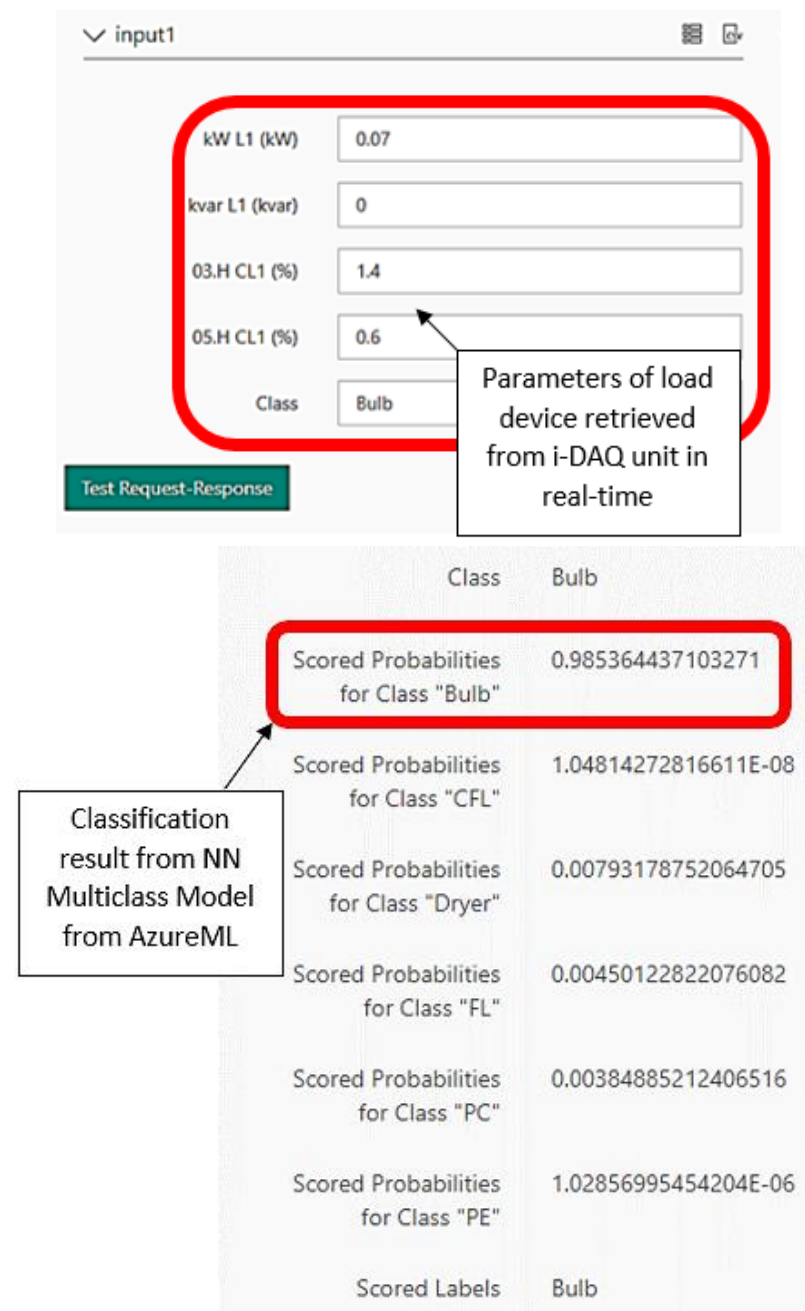

Fig. 10. The classification results from Web Service Output Module

\section{V.CONCLUSION}

This paper presents a proposed convenient and cost-effective way of load monitoring via cloud computing technology for the relevant energy applications. Based on low sampling frequency of power measurements, a simple ANN multiclass algorithm for online appliance classification is presented. Three case studies are examined with different input features, i.e. (i) real power $\mathrm{P}$ only, (ii) real and reactive power $\mathrm{PQ}$, and (iii) power $\mathrm{PQ}$ and harmonics. The results indicate that NN Classifier performs better with additional input features for improved accuracy. Furthermore, easy access of web service API to classification model is demonstrated and further applied in smart grid and smart cities applications such as itemized energy bills and personalized energy saving recommendation, demand side response, energy demand prediction and occupancy monitoring. Cloud-based NILM offers a scalable and nonintrusive real-time energy monitoring solution that can accommodate large number of users. Promising results are from the prototype developed warrant further research. For instances, the use of other ML algorithms to evaluate appliance detection accuracy, and development of graphical user interface (GUI) to accomplish the use of 
information to assist users in energy saving. The collection of cloud data can be further analysed to understand user energy consumption behavior on a larger scale.

\section{ACKNOWLEDGMENT}

This work is fully funded by UCTS Research Grant (Project ID: UCTS/RESEARCH/3/2018/13) of University College of Technology Sarawak. The authors would like to thank the Centre of Research and Development (CRD) of UCTS for the support.

\section{REFERENCES}

1. J. A. Hoyo-Montano, C. A. Pereyda-Pierre, J. M. Tarin-Fontes, and J. N. Leon-Ortega, "Overview of Non-Intrusive Load Monitoring: A way to energy wise consumption," in 2016 13th International Conference on Power Electronics (CIEP), 2016, pp. 221-226.

2. UNFCCC, "Adoption of the Paris agreement. Proposal by the president", Paris Climate Change Conference - November 2015, COP 21, vol. 21932 (December), p. 32, Available at: http://unfccc.int/resource/docs/2015/cop21/eng/109r01.pdf

3. "International Energy Agency and the United Nations Environment Programme (2018): 2018 Global Status Report: towards a zero-emission, efficient and resilient buildings and construction sector."

4. K. K. Kee, B.-Y. L. Simon, and S.-M. W. Peter, "A Holistic Approach for Energy Efficiency at a GBI-Platinum Rated Malaysian University Campus," Adv. Sci. Lett., vol. 24, no. 11, 2018, pp. 8753-8758

5. D. Vine, L. Buys, and P. Morris, "The Effectiveness of Energy Feedback for Conservation and Peak Demand: A Literature Review," Open J. Energy Effic., vol. 02, no. 01, 2013, pp. 7-15.

6. G. W. Hart, "Nonintrusive Appliance Load Monitoring," Proc. IEEE, vol. 80, no. 12,1992 , pp. 1870-1891.

7. A. Zoha, et al., "Non-Intrusive Load Monitoring Approaches for Disaggregated Energy Sensing: A Survey," Sensors, vol. 12, no. 12, 2012, pp. 16838-16866.

8. P. Mell, and T. Grance, "The NIST Definition of Cloud Computing Recommendations of the National Institute of Standards and Technology."

9. A. Oppermann, F. G. Toro, F. Thiel, and J.-P. Seifert, "Secure Cloud Computing: Reference Architecture for Measuring Instrument under Legal Control," Secur. Priv., vol. 1, no. 3, 2018, p. e18.

10. S. Agatonovic-Kustrin, and R. Beresford, "Basic concepts of artificial neural network (ANN) modeling and its application in pharmaceutical research," vol. 22, no. 5, 2000, pp. 717-727.

11. X. Meng, Z. Pian, X. Meng, and Z. Pian, "Theoretical Basis for Intelligent Coordinated Control," Intell. Coord. Control Complex Uncertain Syst. Power Distrib. Netw. Reliab., 2016, pp. 15-50.

12. M. A. Nielsen, "Neural Networks and Deep Learning," 2015. [Online]. Available: http://neuralnetworksanddeeplearning.com/chap2.html.

13. B. Familiar, and J. Barnes, Business in Real-Time. Berkeley, CA: Apress, 2017.

14. K.-K. Kee, and B.-Y. L. Simon, "Cloud-Based IoT Solution for Predictive Modeling of Ship Fuel Consumption," in Proceedings of the 2019 8th International Conference on Software and Computer Applications ICSCA' '19, 2019, pp. 44-49.

15. M. Zorotovich, "Current use cases for machine learning in retail and consumer goods | Blog | Microsoft Azure." [Online]. Available: https://azure.microsoft.com/en-gb/blog/current-use-cases-for-machine-lear ning-in-retail-and-consumer-goods/.

16. D. Starr, "Current use cases for machine learning in healthcare | Blog Microsoft Azure." [Online]. Available: https://azure.microsoft.com/en-us/blog/current-use-cases-for-machine-lear ning-in-healthcare/.

17. "Google Cloud Machine Learning Engine." [Online]. Available: https://cloud.google.com/ml-engine/.

18. "IBM Watson [Online]. Available: https://www.ibm.com/my-en/marketplace/watson-studio.

19. "BigML.com.” [Online]. Available: https://bigml.com/.

20. "Amazon SageMaker on AWS." [Online]. Available: https://aws.amazon.com/sagemaker/.

\section{AUTHORS ProfiLe}

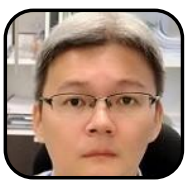

Keh-Kim Kee is a Senior Lecturer of University College of Technology Sarawak (UCTS) and chartered engineer who has registered with Engineering Council of UK (ECUK). Kee is also a senior member of Institute of Electrical \& Electronic Engineers (SMIEEE). His current research interests are artificial intelligence solutions, IoT and smart metering, energy efficiency, and the design of content management systems (CMS) for both commercial and educational sectors.

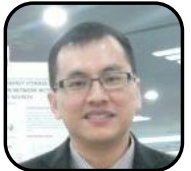

Yun Seng Lim received BEng (Hons) and $\mathrm{PhD}$ from University of Manchester Institute of Science and Technology (UMIST), United Kingdom, in 1998 and 2001 respectively. He is currently a professor of Universiti Tunku Abdul Rahman (UTAR). He is a Professional Engineer with Practising Certificate and CEng registered with Board of Engineer Malaysia and UK Engineering Council respectively. $\mathrm{He}$ is a senior member of IEEE and the Fellow of ASEAN Academy of Engineering and Technology (AAET). As the recipient of the Top Research Scientist Malaysia in 2018, Dr. Lim is also a lead author for the working group III of Intergovernmental Panel on Climate Change (IPCC).

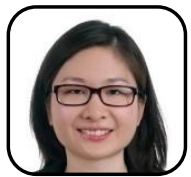

Jianhui Wong received her B.Eng. (Hons) Electrical and Electronic Engineering, MSc. Eng. and Ph.D. Eng. (Electrical) from Universiti Tunku Abdul Rahman (UTAR). She is an Assistant Professor of UTAR, professional engineer registered with the Board of Engineer Malaysia (BEM) and corporate member of the Institute of Engineers Malaysia (IEM). She has actively involved as the principle and co-researcher in the research projects receiving a total amount of RM3 million research grants from various agencies. Her research interests including power system study, energy management and smart grid.

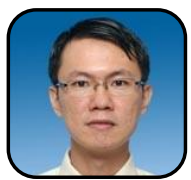

Kein Huat Chua (b. 1979) received the B. Eng. degree in electrical, electronics and system engineering from Universiti Kebangsaan Malaysia, Selangor, Malaysia, in 2004. He received his M. Eng. degree in electrical energy and power system from Universiti Malaya, Kuala Lumpur, Malaysia, in 2008. He received his $\mathrm{PhD}$ in Electrical Engineering from Universiti Tunku Abdul Rahman, Kuala Lumpur, Malaysia. He is currently an Assistant Professor at Universiti Tunku Abdul Rahman. 